\title{
Study of using square-wave coherent method to extracting IP effect
}

\author{
Qiyun Jiang, Dan Mo \\ School of Geo-science and Info-physics, Central South University, Changsha, China \\ 1414250686@qq.com
}

\begin{abstract}
Electromagnetic effect is a major disturbance of measured induced polarization data, and it seriously interferes with the induced polarization measurement and its application effect thus reducing the exploration depth. Therefore, to suppress and eliminate electromagnetic effects effectively is very important. Square-wave coherent method, a unique method to decouple in dual-frequency induced polarization, it is a good way to eliminate the influence of electromagnetic effect from the total field, and at the same time extract the induced polarization effect. Restoring the dual waveform is the key to the square-wave coherent method. When restoring the received waveform to a low-frequency and high-frequency square wave, it is essential to select the inflection point of waves, 0 to 1 or 0 to -1 , which will directly affect the validity of the results of the follow-up process. But due to the interference of electromagnetic effects and ambient noise, it is difficult to pick the inflection point, and which has a bad impact on the promotion and application of the squarewave coherent method. According to the characteristics of inflection point, there's a large difference of amplitude before and after taking off. This article mainly discussed two approaches to pick up take-off points: Based on the amplitude ratio; Base on the amplitude difference. In noisy environments, using these two methods to pick inflection point can still get good results. After finding the inflection point, we can use the squarewave coherent method to calculate the amplitude frequency of induced polarization effect. Compared to the theoretical amplitude frequency, the calculated one, which proves that square-wave coherent method have a good effect in dualfrequency induced polarization measurement.
\end{abstract}

Keywords-dual-frequency induced polarization, square-wave coherent, amplitude ratio, amplitude difference

\section{INTRODUCTION}

Dual-frequency induced polarization method, whose theory is to input two different frequencies into ground and receive field potential-difference information including two main frequencies from underground at the same time ${ }^{[1]}$. It overcomes some shortcomings of traditional variablefrequency method measurement, such as time-consuming operation, inefficiency and its difficulty to improve the accuracy of observation. The dual-frequency induced polarization equipment is light, and easy to operate. Therefore, it has been widely used in sulfide mineral deposits exploration, looking for non-metallic minerals, metal compounds, petroleum, groundwater, and natural gas resources. Electromagnetic coupling is a main interference in frequency domain measurement. So, decoupling is a necessary work. On the one hand, being efficient to remove electromagnetic effect and receive undistorted induced polarization anomalies can evaluate the induced polarization anomaly source nature more accurately, on the other hand, it can improve its prospecting effect and may broaden the application areas. Research how to correct electromagnetic induction coupling has become a hot topic since the 1970s. And it has made some achievements at home and abroad. Like Zonge ${ }^{[2]}$ and Luo, Y.Z. ${ }^{[3]}$ and so on, they proposed some typical methods. But all these methods are indirect, conditional, approximately and need to increase work in the field. When electromagnetic induction coupling is strong and even covers induced polarization effect, they aren't applicable. Square-wave coherent method was proposed by $\mathrm{He}$ J.S. ${ }^{[4]}$ and a lot of quantities prove that the method has a good effect on extracting induced polarization effect while eliminating the effects of electromagnetic coupling for dualfrequency induced polarization measurement.

\section{THEORY AND METHOD}

To make the measured waves coherent with the square wave, in other words, to detect the measured waves which has the same low frequency, same phase or phase difference of $90^{\circ}$ with it, and integral the result, we can obtain the real component $\operatorname{Re}\left(\omega_{\mathrm{L}}\right)$ and the imaginary component $\operatorname{Im}\left(\omega_{\mathrm{L}}\right)$ of the real frequency. Similarly, let square waves, having same high frequency and phase or phase difference of $90^{\circ}$, coherent with measured waves, the real component $\operatorname{Re}\left(\omega_{\mathrm{H}}\right)$ and the imaginary component $\operatorname{Im}\left(\omega_{\mathrm{H}}\right)$ of the high frequency can be available.

Due to the cancellation of positive and negative results, electromagnetic coupling effect is zero after the integration of low frequency, and $\operatorname{Re}\left(\omega_{\mathrm{L}}\right)$ only includes induced polarization effect and primary field while induced polarization effect in $\operatorname{Im}\left(\omega_{\mathrm{L}}\right)$ is very weak. As for real and imaginary components in high frequency, electromagnetic coupling effect is retained completely by integration of $\operatorname{Re}\left(\omega_{\mathrm{H}}\right)$ and $\operatorname{Im}\left(\omega_{\mathrm{H}}\right)$, but induced polarization effect cancel each other mostly.

Because there's no way to calculate theoretical value of electromagnetic coupling effects at present, that can be compared to calculated values by square-wave coherent method. Therefore, in this article, we can only judge the correctness of that method by comparing calculated value of induced polarization effect with theoretical one. Induced polarization effect can be described by real components in low frequency by secondary dual power supply:

$$
F S^{I P}=\frac{\operatorname{Re}\left(\omega_{L_{2}}\right)-\operatorname{Re}\left(\omega_{L_{1}}\right)}{\operatorname{Re}\left(\omega_{L_{1}}\right)} \times 100 \%
$$


This article mainly discussed two approaches to pick up take-off points:

The first one is based on the amplitude ratio. The processing step is to select a time window and slide from scratch, its moving step is set to one generally. At each slide, we need to count the amplitude ratio of the front and rear time window, and then find the maximum value. The point that the maximum amplitude corresponds to in rear time window is just the take-off point we'd like to find;

The second one is to base on the amplitude difference. The slope of a take-off point is the biggest among all points, so let the second one subtract the first one by turn, and then find the maximum amplitude difference between two points and the point with the larger amplitude is what we are looking for.

\section{MODEL DESIGN}

As Fig.1 shows, we used a RLC circuit to simulate the circumstance that electromagnetic effect and induced polarization effect coexist. By changing frequencies of dualfrequency transmitting waveforms, we can receive different amplitude frequencies.

As the most simplified Cole-Cole model, RC circuit can simulate induced polarization when inductance $\mathrm{L}$ is cutting off. And similarly, when capacitance $\mathrm{C}$ gets cutting off, RL circuit can describe electromagnetic induction.

In Fig.1, we use $\mathrm{A}$ and $\mathrm{B}$ to present the transmitting electrodes, while $\mathrm{M}, \mathrm{N}$ to receiving electrode. $\mathrm{R}_{0}$ is dividing resistor. Depending on the model, we obtain theoretical amplitude frequency of square-wave coherence by formula (2)(4), as TABLE I shows.

$$
\begin{gathered}
Z_{M V}=\frac{\left(R_{1}+\frac{1}{j \omega C}\right)\left(R_{2}+j \omega L\right)}{\left(R_{1}+\frac{1}{j \omega C}\right)+\left(R_{2}+j \omega L\right)} \\
U_{U N}=\frac{Z_{M V}}{R_{0}+Z_{U N}} U_{A B} \\
F_{S}=\frac{\left|U_{M N d}\right|-\left|U_{U N G}\right|}{\left|U_{U N G}\right|} \times 100 \%
\end{gathered}
$$

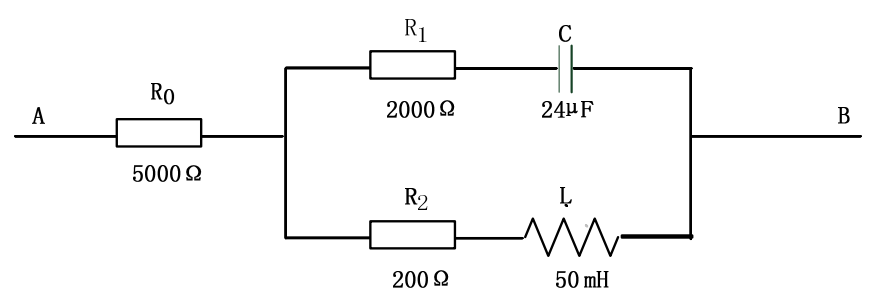

Fig.1. Circuit schematic included IP and EM effects.

\section{Measured Data Processing}

Using the dual-frequency wave in zero audio group ( $\mathrm{fg}=$ $1 \mathrm{~Hz}$ and $\mathrm{fl}=1 / 13 \mathrm{~Hz}$ ) and 3 audio group $(\mathrm{fg}=8 \mathrm{~Hz}$ and $\mathrm{fl}=8$ $/ 13 \mathrm{~Hz}$ ) to acquire data. In the induced polarization survey, when $\mathrm{Fs} \leq 2 \% \sim 4 \%$, we can apply the mean square error $\varepsilon_{\text {to }}$ measure observation accuracy:

$$
\varepsilon= \pm \sqrt{\frac{\left(F_{S}-F_{S}\right)^{2}}{2}}
$$

Where Fs' is the theoretical amplitude frequency, Fs is the measured amplitude frequency. Under normal circumstances, is credible.

TABLE I. THEORETICAL AMPLITUDE FREQUENCY

\begin{tabular}{|c|c|c|c|c|}
\cline { 1 - 4 } High-frequency & \multirow{2}{*}{$\mathbf{1} \mathbf{1 3 H z}$} & $\mathbf{2 / 1 3 H z}$ & $\mathbf{4 / 1 3 H z}$ & $\mathbf{8} / \mathbf{1 3 H z}$ \\
\cline { 1 - 4 } Low- frequency & & & & \\
\hline $1 / 13 \mathrm{~Hz}$ & - & 0.0161 & 0.0802 & 0.328 \\
\hline $2 / 13 \mathrm{~Hz}$ & - & - & 0.064 & 0.3118 \\
\hline $4 / 13 \mathrm{~Hz}$ & - & - & - & 0.2476 \\
\hline $8 / 13 \mathrm{~Hz}$ & - & - & - & - \\
\hline
\end{tabular}

After finding each take-off point of the measured waveform, we can restore the dual waveforms. Zero frequency group data, for example, shown in Fig.2. The sample rate of measured waves is $2000 \mathrm{~Hz}$.
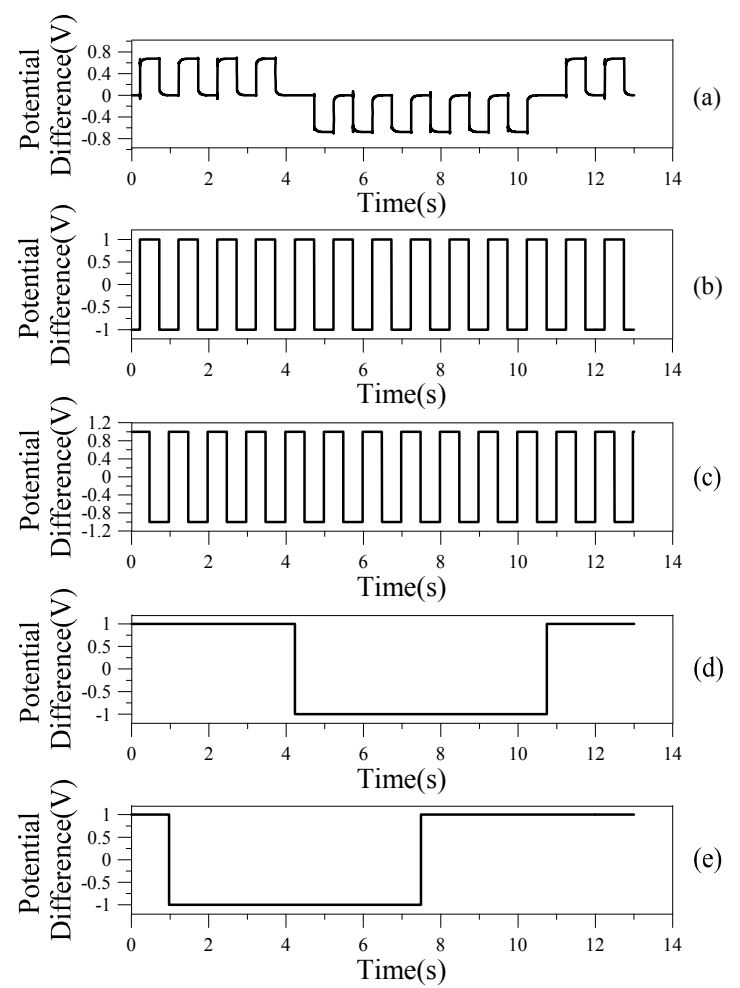

Fig.2. (a) Measured waves in zero frequency group; (b)High-frequency real components with same frequency and phase;(c) High-frequency real components with same frequency and phase difference of $90^{\circ}$; (d)Lowfrequency real components with same frequency and phase;(e) Low-frequency real components with same frequency and phase difference of $90^{\circ}$; 

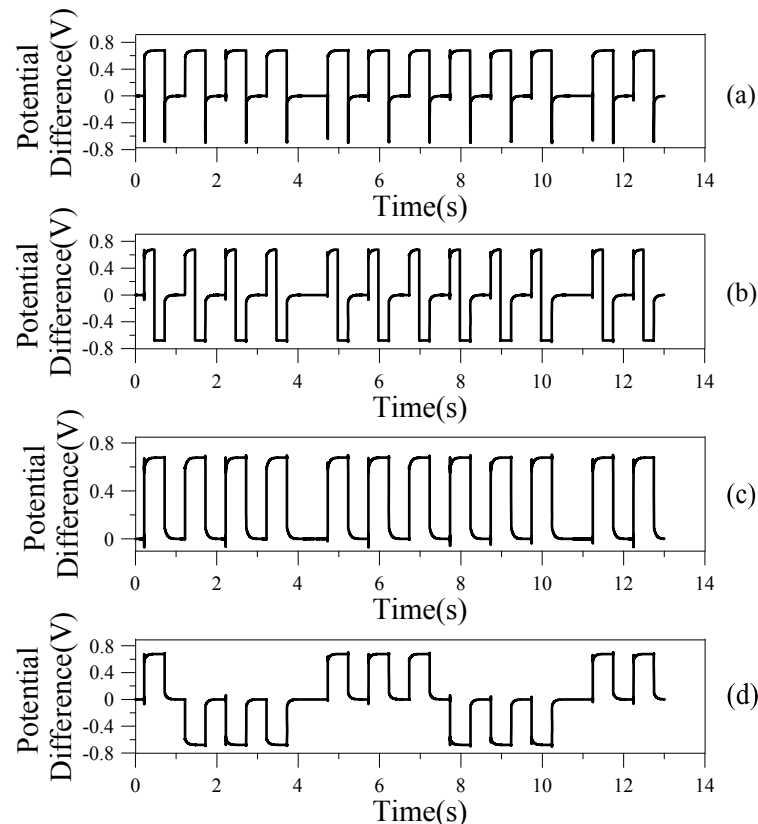

(a)

Fig.3. (a) Restored high-frequency real components with same frequency and phase;(b) Restored high-frequency real components with same frequency and phase difference of $90^{\circ}$; (c) Restored low-frequency real components with same frequency and phase;(d) Restored low-frequency real components with same frequency and phase difference of $90^{\circ}$;

Letting the stored high-frequency and low-frequency waveforms be coherent with measured waveforms, as shown in Fig.3. After integrated, we can obtain the result as follows:

$$
\begin{aligned}
& \operatorname{Re}\left(\frac{1}{13} H Z\right)=4.0880, \operatorname{Im}\left(\frac{1}{13} H Z\right)=-3.1733 \times 10^{-4}, \\
& \operatorname{Re}\left(\frac{8}{13} H Z\right)=4.0744, \operatorname{Im}\left(\frac{8}{13} H Z\right)=-0.0929
\end{aligned}
$$

By formula (1), the amplitude-frequency IP effect is calculated as follows:

$$
F_{S}^{I P}=\frac{\operatorname{Re}\left(\frac{1}{13}\right)-\operatorname{Re}\left(\frac{8}{13}\right)}{\operatorname{Re}\left(\frac{8}{13}\right)} \times 100 \%=0.3338 \%
$$

Due to the model, as Fig.1 shows, the value of theoretical amplitude frequency IP is $0.3280 \%$, then, by formula (5), the mean square error is calculated as follows:

$$
\varepsilon \approx \sqrt{\frac{\left(F_{S}-F_{S}^{\prime}\right)^{2}}{2}}=\sqrt{\frac{(0.3338 \%-0.3280 \%)^{2}}{2}}=0.0041 \%
$$

In order to study the two anti-jamming capabilities, we added a group of Gaussian noise to the measured data. To pick up the first take-off point of two sets of data collected, for example, as shown in Table II. Amplitude ratio method to pick off point locations is recorded as 1 , while demand amplitude difference is 2 .
TABLE II. INFLECTION POINT POSITION COMPARISON IN NOISE ENVIRONMENTS

\begin{tabular}{|c|c|c|c|c|}
\hline \multirow{2}{*}{ SNR (db) } & \multicolumn{2}{|c|}{ 0 audio group } & \multicolumn{2}{c|}{ 2 audio group } \\
\cline { 2 - 5 } & 1 & 2 & 1 & 2 \\
\hline 70 & 433 & 433 & 236 & 236 \\
\hline 60 & 433 & 433 & 236 & 236 \\
\hline 50 & 433 & 433 & 236 & 236 \\
\hline 40 & 433 & 433 & 236 & 236 \\
\hline 20 & 433 & 433 & 236 & 236 \\
\hline 0 & 433 & 433 & 236 & 236 \\
\hline
\end{tabular}

As it can be seen from TABLE II, although the noise level increases, but the use of these two methods can still pick out the exact take-off point accurately. It's necessary to choose a smaller window width under the circumstance of low SNR.

\section{CONCLUSION}

The results proves that the method picking up take-off point automatically has a strong anti-interference ability, and can exclude the human induced errors factors. Probably because of the instrument itself, or electromagnetic coupling, the measured waveform width is not equidistant, which caused that integrating the real and imaginary components in low frequency cannot completely suppress EM effects, which will bring the error results. Therefore, just to pick the first inflection point is not enough, we need to pick up all take-off points. Because that the frequency difference between low frequency square wave of two dual-frequency waves is small, resulting in the calculated amplitude frequency is small, the actual work is very susceptible to outside interference. Therefore, a lot of work needs further study.

\section{AKNOWLEDGEMENT}

We are indebted to Academician He J.S. for fruitful discussions. D.M. thanks Dr Jiang Q.Y. for his instruction and encouragement.

\section{REFERENCES}

[1] He. J.S., Dual-frequency induced polarization method, Beijing: Higher Education Press, 2006.

[2] Zonge K L, Wynn J C. "Recent advances and applications in complex resistivity measurements," Geophysics, 1975, pp.851-864.

[3] He. J.S., Wang. S.W. and Tang. J.T., "Square-wave coherent method extracting IP effect directly," Chinese Journal of Geophysics, 1994, pp.516-523.

[4] Zhang. W., Wang. H., Li H.C., Wang. Y.C., and Duan.Y.Q, ”Using the transform window statistical energy ratio method to pick up early seismic waves," Geophysical and Geochemical Exploration, 2009, pp. $178-180$ 